\title{
Sistemas Tutores Inteligentes voltados ao apoio da escrita acadêmica: uma revisão sistemática
}

\section{Intelligent Tutoring Systems applied to academic writing: a systematic review}

\author{
Ederson Bastiani \\ Universidade Federal do Rio Grande do Sul \\ Alex Eder da Rocha Mazzuco \\ Universidade Federal do Rio Grande do Sul \\ Eliseo Berni Reategui \\ Universidade Federal do Rio Grande do Sul
}

\begin{abstract}
Resumo: As habilidades de escrita representam uma parte crítica da comunicação durante a trajetória acadêmica dos estudantes, uma vez que trata de uma competência imprescindível aos estudantes, pois é por meio dela que ocorre a socialização e o reconhecimento dos discentes em suas áreas. Sabe-se, no entanto, que a produção da escrita acadêmica não é uma tarefa fácil. Neste sentido, este artigo apresenta uma revisão sistemática da literatura que busca identificar os estudos relevantes no que tange à utilização da tecnologia conhecida como sistema tutor inteligente como forma de apoio à escrita acadêmica. Esta revisão foi fundamentada nas diretrizes propostas por Kitchenham, contemplando buscas em repositórios científicos relevantes. Foram encontradas lacunas capazes de representar um campo fértil de pesquisa e desenvolvimento, como a ausência de aplicação de sistemas tutores inteligentes para auxiliar a escrita de projetos de pesquisa.
\end{abstract}

Palavras-chave: Escrita acadêmica; Sistemas tutores inteligentes; Revisão sistemática da literatura.

\begin{abstract}
Writing skills represent a critical part of communication during the students' academic trajectory, since it is an essential competence for students, as it is through it that socialization and recognition of students in their areas. It is known, however, that the production of academic writing is not an easy task. In this sense, this article presents a systematic review of the literature that seeks to identify the relevant studies regarding the use of technology known as the intelligent tutoring system as a way of supporting academic writing. This review, which was based on the guidelines proposed by Kitchenham and carried out with searches in relevant scientific repositories. We found gaps that may represent a fertile field of research and development, such as the absence of the application of intelligent tutoring systems to assist the writing of research projects.
\end{abstract}

Keywords: Academic writing; Intelligent tutoring systems; Systematic literature review.

BASTIANI, Ederson; MAZZUCO, Alex Eder da Rocha; REATEGUI, Eliseo Berni. Sistemas Tutores Inteligentes voltados ao apoio da éscrita acádêmica: uma revisão sistemática. Informática na Educação: teoria \& prática, Porto Alegre, v. 23 , n. 3 ,
p. $85-99$, set./dez. 2020 . 


\section{Introdução}

A escrita é uma das formas mais antigas de representação da comunicação e a sua importância como evolução da humanidade é algo indiscutível (REATEGUI et al., 2014). Consiste em um instrumento complexo para o desenvolvimento da consciência, possibilitando ao ser humano reger sua própria prática intelectual e, por ser uma forma de linguagem que opera na concepção e na ampliação das funções psíquicas superiores, demanda uma natureza de abstração distinta da linguagem oral (FARIAS; BORTOLANZA, 2015). Esta abstração, exigida pela escrita, permite ao homem, como ser integrante e alicerçador de uma coletividade maior, interagir, expressar e registrar sentimentos, opiniões e inspirações, por meio da redação diária, como notas, mensagens de e-mail e postagens em redes sociais (WESTON-SEMENTELLI; ALLEN; MCNAMARA, 2018).

No meio acadêmico não é diferente, neste caso, a escrita, agora dita acadêmica (ou científica), está presente em todos os níveis de educação e representa o maior componente de ensino e aprendizagem da vida escolar (STROBL et al., 2019). Assim, caracteriza-se por ser uma competência associada ao sucesso, tanto no que se refere ao bem-estar do aluno quanto à sua permanência e seu êxito. No contexto do ensino superior, a produção textual é uma prática rotineira e exigida por todos os cursos. Trata-se de uma habilidade imprescindível aos estudantes, uma vez que é por meio dela que ocorre a socialização e o reconhecimento dos discentes em suas áreas. Neste sentido, os estudantes produzem textos de acordo com diversos gêneros acadêmicos, nos quais devem demonstrar sua proficiência de escrita (ARAÚJO; DIEB; COSTA, 2017).

O processo de escrita acadêmica, entretanto, é uma tarefa desafiadora, sendo notórias as dificuldades apresentadas pelos alunos para organizar, escrever e revisar os seus textos. Neste sentido, é necessário considerar que os primeiros contatos dos estudantes com a escrita científica ocorrem no ambiente acadêmico, onde grande parte deles apresenta significativa inexperiência quanto aos gêneros, procedimentos e convenções acadêmicas (LI et al., 2018) (RAPP; KAUF, 2018). Desta forma, no momento em que o aluno confronta-se com a necessidade de construir um texto acadêmico, ele percebe que esta atividade demanda conhecimentos mais específicos e aprofundados do que a escrita cotidiana (LIN; LIU; WANG, 2017) (LI et al., 2018).

Para Rigo et al. (2018), os momentos de escrita acadêmica repercutem de diferentes formas para os discentes, como sentimentos de insegurança e ansiedade. Tais sentimentos evidenciamse no momento em que os estudantes expressam seus questionamentos, por exemplo, "Como faço para iniciar uma escrita?" ou "O que eu faço para escrever melhor?". Logo, percebe-se que o exercício de conectar o pensamento ao que se quer expressar tende a ser um processo complexo (REATEGUI; OLIVEIRA, 2017). Assis (2014) considera que fatores como a inexperiência com relação às convenções acadêmicas e à heterogeneidade, tanto cultural quanto de trajetória escolar da população discente, são oriundos do processo recente de massificação do ensino superior. Ainda, segundo a autora, estes problemas são capazes de gerar entraves que, em alguns casos, mantêm-se durante toda a vida acadêmica, inclusive após a graduação. 
De forma a minimizar estas lacunas, inúmeros cursos de nível superior têm estimulado a inclusão de disciplinas nos semestres iniciais com a intenção de promover a aquisição de habilidades de escrita, o que, segundo Allen et al. (2016) é possível por meio de estratégias instrucionais, juntamente com a prática extensiva e feedback individualizado fornecido aos estudantes durante o processo de escrita. Todavia, é necessário, também, considerar a perspectiva do professor. Por esse prisma, o ensino dos gêneros acadêmicos implica em uma tarefa que exige tanto habilidades no domínio técnico, quanto didático, para apresentar aos alunos o raciocínio que permeia o processo de escrita (ARAÚJO; DIEB; COSTA, 2017).

Desta maneira, a metodologia que se observa no cotidiano é baseada em atividades que iniciam pela escrita de um rascunho e, que, então, é aprimorado após as recomendações de um professor (revisor, orientador ou tutor), em um processo interativo de geração, organização e refinamento de ideias (LÓPEZ; BETHARD; LÓPEZ-LÓPEZ, 2014). Na prática, porém, os professores raramente dispõem da quantidade de tempo necessária para planejar o processo, elaborar materiais de instrução, ler, editar e proporcionar a devolutiva adequada aos alunos. Assim, torna-se relevante o estudo e a utilização de tecnologias que tenham potencial para qualificar o processo da escrita acadêmica.

Embora pesquisadores tenham aumentado seus esforços na busca do desenvolvimento de soluções computacionais, a maioria das pesquisas conduzidas sobre este assunto tem focado em fornecer avaliações sobre os textos acadêmicos, e não no processo de escrita (ALLEN et al., 2016a) (STROBL et al., 2019). Nesse sentido, tecnologias baseadas em Sistemas Tutores Inteligentes (STIs) podem ser aplicadas.

Sistemas tutores inteligentes podem ser entendidos como programas de computador que incorporam técnicas de Inteligência Artificial (IA) para possibilitar a concepção de tutores que sabem o que, a quem e como ensinar (NWANA, 1990). Da mesma forma que a área da inteligência artificial busca reproduzir no computador um comportamento que, se fosse realizado por um humano, seria considerado inteligente, um STI tenta reproduzir um comportamento em computador que, se realizado por um professor, seria descrito como "bom tutor", apresentando aspectos como tutoria individualizada e feedback imediato para os estudantes. Isso ocorre por meio da interseção das áreas da Educação, Psicologia e Ciência da Computação. Enquanto a Computação, através técnicas de IA, fornece a "inteligência" relacionada ao ensino, a área da Psicologia contribui com modelos sobre como as pessoas pensam e constroem conhecimento. Soma-se a estas duas a área da Educação, a qual foca em apoiar de forma efetiva o processo de ensino-aprendizagem (HIEU; MUSTAPHA, 2018).

Por serem capazes de monitorar as atividades desenvolvidas pelos estudantes, os STIs possuem subsídios para realizar inferências com relação às fraquezas ou às habilidades dos estudantes e, desta forma, sugerirem recomendações e materiais de apoio. Além disso, os STIs são sistemas adaptativos, capazes de se ajustar e propiciar respostas aos alunos que contenham tarefas ou instruções apropriadas a cada perfil de aluno com ausência de intervenção humana (ALLEN et al., 2016a). 
A partir das necessidades relacionadas à investigação de como apoiar os acadêmicos na correta construção da escrita científica e das possibilidades apresentadas pelos STIs, este artigo apresenta o resultado uma revisão sistemática da literatura com o objetivo de identificar o que tem sido desenvolvido envolvendo esta temática.

O artigo está estruturado da seguinte forma: a seção 2 apresenta os materiais e métodos utilizados. A seguir, na seção 3, os resultados desta revisão sistemática são discutidos. Por fim, apresentam-se as considerações finais, na seção 4, e as referências bibliográficas que suportaram a pesquisa.

\section{Metodologia}

O propósito deste trabalho é identificar e analisar os trabalhos relevantes relacionados à utilização de sistemas tutores inteligentes como forma de apoio à escrita acadêmica. Para isso, foi realizada uma revisão sistemática da literatura fundamentada nas diretrizes propostas por Kitchenham (2004), as quais contemplam as fases de: (a) planejamento, onde são definidas as questões de pesquisa, os termos e a estratégia de busca, bem como os critérios de inclusão, de exclusão e de qualidade; (b) a condução, onde ocorre a submissão dos termos de busca às bases, seleção dos artigos primários, avaliação da qualidade e extração de dados; e (c) análise dos resultados, etapa na qual os dados são sintetizados e consolidados de forma a responderem às questões de pesquisa. A Figura 1 apresenta graficamente a metodologia adotada para esta revisão.

Figura 1 - Metodologia aplicada

\section{Planejamento}

- Questões de pesquisa

- Estratégia de busca

- Critérios de inclusão, de exclusão e de qualidade

\section{Condução}

- Atividade de busca

- Seleção de estudos primários

- Avaliação de qualidade

- Extração de dados
Análise dos resultados

- Sintetização

Fonte: Adaptado de Kitchenham (2004).

Como etapa inicial do planejamento, definiu-se que este estudo buscaria responder a seguinte questão de pesquisa (QP): "Como os sistemas tutores inteligentes têm contribuído no processo de escrita acadêmica?". A partir da QP, outras quatro questões secundárias foram definidas como relevantes, as quais são apresentadas na Tabela 1.

Tabela 1 - Questões de pesquisa secundárias

\begin{tabular}{|c|c|}
\hline Código & Enunciado \\
\hline Q1 & Para quais gêneros de escrita acadêmica os trabalhos são direcionados? \\
\hline Q2 & Qual é o público-alvo dos estudos? \\
\hline Q3 & Quais são os objetivos dos trabalhos? \\
\hline
\end{tabular}




\begin{tabular}{|l|l|}
\hline Q4 & Quais aspectos linguísticos da escrita acadêmica são abordados? \\
\hline
\end{tabular}

Fonte: Os autores.

De forma a encontrar trabalhos que pudessem ser relevantes para este estudo, utilizaram-se os seguintes termos (string de busca): "intelligent AND tutor* AND system* AND (academic OR scientific) AND writ*". Nesta string, o caractere "*" tem o sentido de representar as variações que possam ocorrer em função do radical de cada termo, por exemplo, tutor e tutorING.

Na sequência, procedeu-se a submissão da string aos mecanismos de busca das bases de dados Scopus, IEEEXplore e ACM Digital Library, devido à relevância acadêmica destes repositórios. Configurou-se cada mecanismo de busca para pesquisar artigos acadêmicos considerando o título e abstract dos trabalhos publicados nos a partir do ano de 2014. Este processo de busca foi supervisionado por três pesquisadores.

Após o processo de busca, os documentos obtidos foram submetidos aos critérios de exclusão (CEs), os quais possuem o objetivo de eliminar os trabalhos que não condizem com o escopo desta revisão. Assim, os critérios excludentes de estudos primários são apresentados na Tabela 2.

Tabela 2 - Critérios de exclusão

\begin{tabular}{|c|c|}
\hline Código & Critério de exclusão \\
\hline CE1 & Trabalhos que apresentam revisões, mapeamentos sistemáticos ou surveys. \\
\hline CE2 & Trabalhos escritos em idioma diferente do Inglês. \\
\hline CE3 & $\begin{array}{c}\text { Trabalhos que não apresentam os termos de busca no título ou no abstract ou } \\
\text { que não sejam relacionados com a escrita acadêmica. }\end{array}$ \\
\hline CE4 & Trabalhos que fossem duplicados ou versões anteriores de um mesmo projeto. \\
\hline
\end{tabular}

Fonte: os autores.

Do mesmo modo, estabeleceram-se critérios de qualidade para que um trabalho fosse considerado relevante, dessa forma, a obra deveria responder de forma positiva a, pelo menos, três dos seguintes questionamentos que constam na Tabela 3.

Tabela 3 - Critérios de avaliação de qualidade

\begin{tabular}{|c|c|}
\hline Sigla & Critério de avaliação de qualidade \\
\hline AQ1 & O trabalho apresenta a justificativa do estudo? \\
\hline AQ2 & O trabalho detalha os materiais e métodos utilizados? \\
\hline AQ3 & O trabalho aborda com clareza qual tipo de texto acadêmico é estudado? \\
\hline AQ4 & O trabalho informa qual o nível de abrangência da escrita é proposto? \\
\hline AQ5 & O trabalho relata com clareza quais foram os resultados alcançados? \\
\hline
\end{tabular}

Fonte: os autores.

Após a seleção dos artigos, considerando os critérios de exclusão e de qualidade, criou-se uma tabela para extração dos seguintes dados, capazes de auxiliar nas respostas às questões norteadoras do projeto: finalidade do trabalho, gênero de escrita acadêmica, abrangência de cobertura dos textos, público-alvo, técnicas e tecnologias utilizadas e elementos textuais abordados. 
Por fim, procedeu-se a síntese de informações e análise dos resultados obtidos neste estudo, apresentados na seção 3 .

\section{Resultados da Pesquisa}

A partir da estratégia de busca definida neste estudo, foram encontrados 24 documentos, dos quais 1 documento foi excluído da análise de revisão por se tratar de uma edição de anais de evento e não de material primário. A Figura 2 apresenta a distribuição dos 23 documentos restantes em função do ano de publicação.

Figura 2 - Número de artigos agrupados por ano de publicação

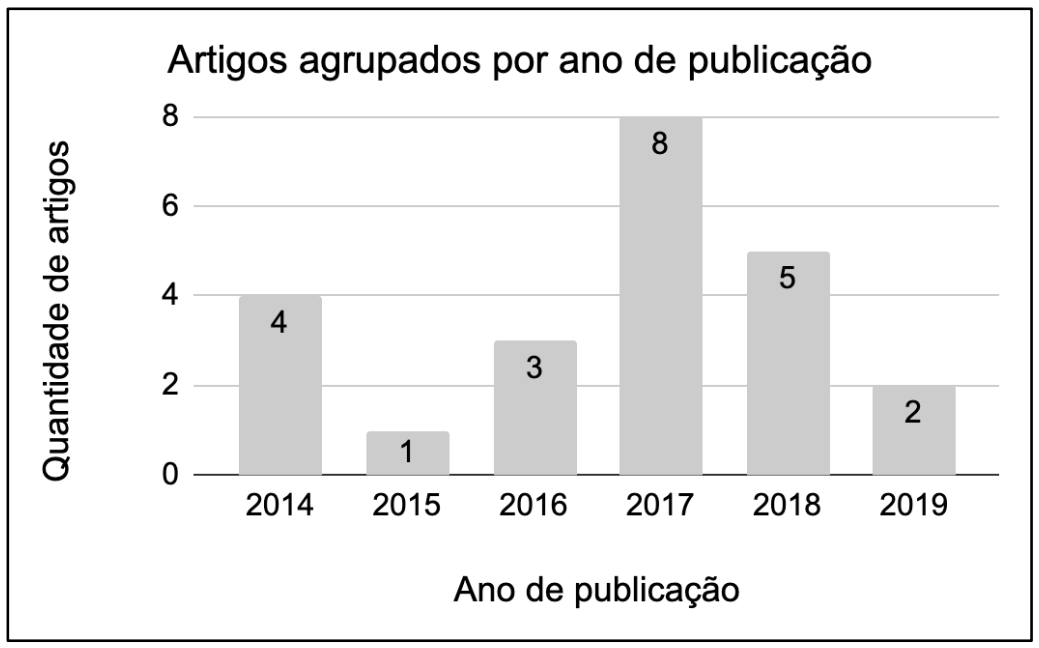

Fonte: os autores.

Em seguida, realizou-se a leitura dos títulos e abstracts de todos os trabalhos para identificar os estudos candidatos a serem analisados, os quais foram submetidos aos CEs. A Figura 3 apresenta a aplicação dos critérios de exclusão, no entanto, é importante ressaltar que um documento pode ser considerado excluído a partir de mais de um CE.

Figura 3 - Aplicabilidade dos critérios de exclusão

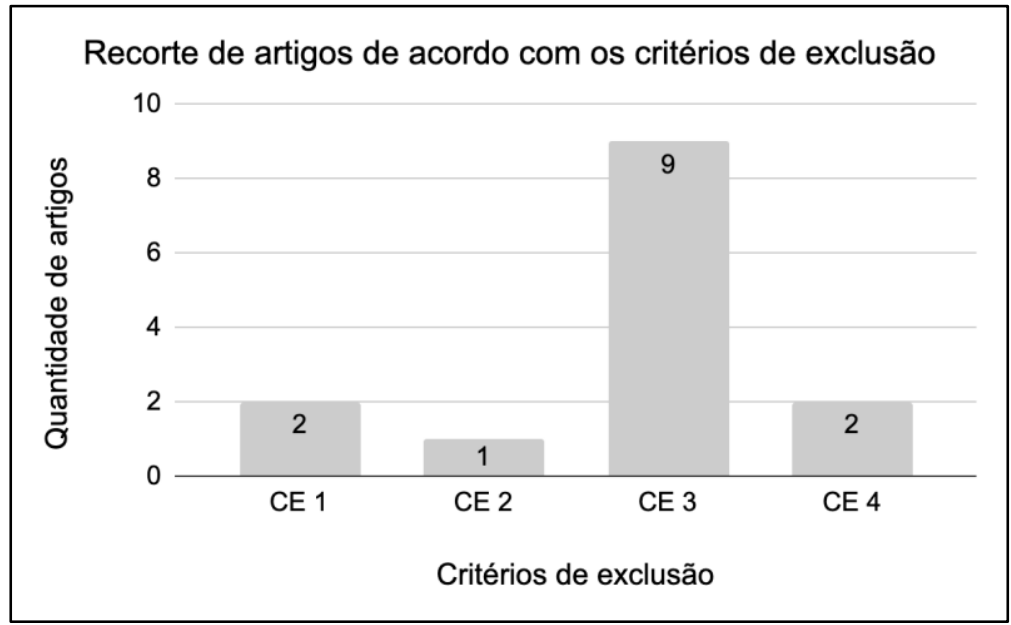


Fonte: os autores.

Ao todo, 14 documentos atenderam a critérios de exclusão, sendo que: 14,28\% dos documentos caracterizavam-se como revisões da literatura; 7,14\% encontravam-se escritos em idioma discordante da língua inglesa; $64,28 \%$ apresentam trabalhos cujo foco incompatibilizavase com a escrita acadêmica; e, 14,28\% consistiam-se em trabalhos duplicados, ou seja, foram encontrados em mais de uma base de dados.

Para o conjunto dos demais 6 trabalhos, procedeu-se a leitura na íntegra, de forma a permitir a avaliação da qualidade dos estudos. Nesse ponto, todos os trabalhos atenderam aos critérios de qualidade estabelecidos. A Tabela 3 apresenta a relação de trabalhos remanescentes neste estudo.

Tabela 3 - Lista dos artigos aceitos

\begin{tabular}{|l|c|}
\hline \multicolumn{1}{|c|}{ Referência } & Título do trabalho \\
\hline (LÓPEZ; LÓPEZ-LÓPEZ, 2014) & $\begin{array}{c}\text { Mining domain knowledge for coherence assessment } \\
\text { of students proposal drafts. }\end{array}$ \\
\hline $\begin{array}{l}\text { (LÓPEZ; BETHARD; LÓPEZ-LÓPEZ, } \\
\text { 2014) }\end{array}$ & $\begin{array}{c}\text { Identifying Weak Sentences in student drafts: } A \\
\text { tutoring system. }\end{array}$ \\
\hline (ALLEN et al., 2016) & $\begin{array}{c}\text { Investigating boredom and engagement during writing } \\
\text { using multiple sources of information: } \text { The essay, the } \\
\text { writer, and keystrokes. }\end{array}$ \\
\hline $\begin{array}{l}\text { (GARCÍA-GORROSTIETA; LÓPEZ- } \\
\text { LÓPEZ; GONZÁLEZ-LÓPEZ, 2017) }\end{array}$ & $\begin{array}{c}\text { Towards automatic assessment of argumentation in } \\
\text { theses justifications. }\end{array}$ \\
\hline (LIN; LIU; WANG, 2017) & $\begin{array}{c}\text { Development and usability test of an e-learning tool } \\
\text { for engineering graduates to develop academic writing } \\
\text { in English: } \text { A case study. }\end{array}$ \\
\hline (LI; GOBERT; DICKER, 2017a) & $\begin{array}{c}\text { Dusting Off the Messy Middle: Assessing Students' } \\
\text { Inquiry Skills Through Doing and Writing. }\end{array}$ \\
\hline (LI et al., 2018) & $\begin{array}{c}\text { Students' academic language use when constructing } \\
\text { scientific explanations in an intelligent tutoring } \\
\text { system. }\end{array}$ \\
\hline (RAPP; KAUF, 2018) & $\begin{array}{c}\text { Scaling Academic Writing Instruction: Evaluation of a } \\
\text { Scaffolding Tool (Thesis Writer) }\end{array}$ \\
\hline
\end{tabular}

Fonte: os autores.

\section{Análise e Discussão dos Trabalhos Encontrados}

Embora existam poucos estudos associando os tópicos de sistemas tutores inteligentes e escrita acadêmica, percebe-se, a partir da seleção de trabalhos, que os pesquisadores têm buscado atender tanto problemas específicos da escrita, como quanto os textos de uma forma geral. Porém, grande parte dos trabalhos foca no desenvolvimento de técnicas e ferramentas para avaliação da escrita, e não no apoio ao seu processo.

No trabalho de López e López-López (2014) os autores apresentam a proposta para um módulo analisador de coerência textual como parte de um STI. Este módulo utiliza a técnica de análise semântica latente (LSA) para analisar a coerência global entre sete seções em uma proposta de tese: definição do problema, justificativa, objetivo, questões de pesquisa, hipótese, metodologia e conclusão. Para avaliar a proposta, os autores realizaram dois experimentos. 0 primeiro, buscou encontrar os espaços semânticos dentro de cada seção de um projeto, a partir de um corpus de conhecimento composto por 410 exemplos retirados de projetos de pesquisa 
da área da informática, onde cada seção destes projetos foi anotada por 3 revisores humanos e depois processada computacionalmente. Os resultados, então, foram comparados estatisticamente. $O$ segundo procedimento procurou identificar o grau de relação entre as seções do texto, ou seja, a relação de coerência global. Ao submeter um texto ao STI, a análise da coerência era realizada e indicada em três níveis: baixa, média e alta, informando, também, se algum conceito seria externo à área da ciência da computação. Segundo os autores, esta avaliação de coerência é a primeira a explorar os recursos existentes por seções específicas para a ciência da computação e a tecnologia da informação. O experimento, entretanto, não foi testado com estudantes.

Outro estudo publicado por López, Bethard e López-López (2014), apresenta um componente para um STI responsável por identificar sentenças fracas em conclusões de teses. Para os autores, uma seção de conclusão de tese é considerada forte (aceitável) se fornece: análise do cumprimento dos objetivos da pesquisa; uma resposta à questão de pesquisa geral; e um julgamento de valor dos resultados. Ainda, deve evitar termos gerais e palavras especulativas, utilizando termos específicos de determinada área. Nesse sentido, o STI busca classificar os parágrafos da seção de por meio de análise da riqueza lexical, medida de similaridade entre sentenças, uso de termos especulativos e sobreposição com termos das seções de conclusão de teses aprovadas e avaliadas por especialistas. Para o estudo, foram utilizadas 544 sentenças de 55 teses de Ciência da Computação, as quais foram anotadas por especialistas humanos e, posteriormente, submetidas a algoritmos baseados em processamento de linguagem natural para geração de um modelo computacional. Apesar dos benefícios do estudo, não foram realizados procedimentos com estudantes.

Para García-gorrostieta; López-López; González-López (2017), a argumentação durante do processo de escrita acadêmica é apontada como uma habilidade crítica durante a vida acadêmica. No estudo, os autores apresentam o desenvolvimento e um experimento realizado com um módulo de argumentação do TURET (Tutor for Thesis Writing), um sistema online voltado à escrita acadêmica. $O$ objetivo deste módulo é realizar a identificação e a avaliação de parágrafos argumentativos na justificativa de teses e fornecer feedback formativo para esta avaliação. Um experimento contendo 232 parágrafos de justificativas em teses foi realizado para testes, onde os resultados apontaram que o sistema é capaz de identificar parágrafos que não possuem argumentos, permitindo que os estudantes e orientadores possam realizar revisões para melhoria da escrita nesse sentido. Nenhum experimento, entretanto, foi realizado diretamente com estudantes e, desta forma, não é possível identificar os ganhos reais com relação à escrita acadêmica.

Já no trabalho de Allen et al. (2016a) os autores analisam se índices coletados por um sistema tutor inteligente, como propriedades linguísticas e semânticas do texto gerado e dados de digitação, podem ser utilizados para modelar estados afetivos e, a partir disso, gerar feedbacks mais individualizados aos alunos. Segundo os autores, grande parte dos sistemas desenvolvidos para prática de escrita tem focado na avaliação da escrita, deixando lacunas abertas em questões como individualização e instruções adaptadas. Ainda, a maioria das avaliações tem sido de 
caráter somativo. Em face disso, o trabalho apresenta o Writing-Pal, um STI desenvolvido para em fornecer estratégias instrucionais e práticas de escrita para estudantes do ensino médio e universitários em anos iniciais. O sistema auxilia os estudantes durante as três fases do processo de escrita: pré-escrita, escrita e revisão. Cada uma destas etapas é apoiada por lições em vídeo, narradas por um agente pedagógico animado. Após assistir uma lição, os estudantes podem utilizar mini jogos para avaliar seu aprendizado. Outra funcionalidade relevante do Writing-Pal é a avaliação automática dos textos por meio de uma variedade de aspectos linguísticos, a qual fornece tanto feedback formativo, quanto somativo. Um conjunto de 44 estudantes de graduação participou do estudo. Em tal experimento os alunos foram monitorados no decorrer do processo de escrita de um ensaio durante 30 minutos. Os resultados sugeriram que os logs, propriedades de texto e outros índices mensurados podem ser utilizados para desenvolver modelos de estados afetivos dos alunos ao longo das sessões de redação, possibilitando, assim, o desenvolvimento de sistemas mais adaptativos ao suporte da escrita acadêmica.

No trabalho de Lin, Liu e Wang (2017), os autores argumentam sobre a necessidade dos estudantes, não falantes da língua inglesa, para publicar artigos científicos em inglês. Segundo os autores, existem vários projetos que buscam melhorar a proficiência de escrita dos alunos utilizando e-learning, porém pouca atenção tem sido dada à escrita acadêmica. Baseados nessa problemática, os autores apresentam a implementação e avaliação de uma ferramenta, nomeada EEJP, que oferece instruções de escrita baseada em gênero (GBWI - Genre-based writing instructions) para o desenvolvimento de habilidades de escrita em artigos científicos de estudantes de pós-graduação falantes de mandarim como primeira língua. O sistema é basicamente composto por três módulos nomeados de My Paper, References and Notes e Introduction to Journal Paper Writing and Format. Estes módulos permitem que os usuários cadastrem informações como nomes dos autores, evento ou periódico para o qual o trabalho será submetido, e gerenciem referências e áreas para escrita. O sistema também disponibiliza ferramentas como dicionário, exemplos de sentenças e explicações para o aprendizado da escrita acadêmica de forma geral, ou seja, o trabalho não é focado em aspectos específicos da escrita. A fim de validar a proposta, um estudo de caso foi desenvolvido pelos autores, no qual 32 estudantes ( 14 de doutorado e 18 de mestrado) participaram de uma atividade de escrita por um período de 4 horas e, logo após, instigados a responderem um questionário avaliativo. Os resultados mostraram que o GBWI pode ser uma abordagem eficaz de ensino e aprendizagem para educadores e alunos. Nesse sentido, as instruções de estruturas retóricas apresentadas pelo sistema, juntamente com os exemplos de frases foram consideradas a parte mais útil do EEJP.

O estudo de Li, Gobert e Dicker (2017b) buscou examinar se as habilidades de investigação dos alunos do ensino fundamental para projetar e conduzir experimento científicos refletem seus desempenhos ao escrever explicações científicas no sistema Inq-ITS, um sistema tutor inteligente voltado a laboratórios virtuais para o ensino de ciências. A motivação para o estudo, segundo os autores, é que as práticas investigativas podem ser divididas em duas categorias: fazer (doing) e escrever explicações científicas (writing). Um experimento foi realizado com 293 estudantes de 18 turmas de uma escola pública a partir de um laboratório virtual para investigar 
o comportamento sobre a densidade de diferentes substâncias, com diferentes quantidades, armazenadas em repositórios distintos. Os testes realizados indicaram que, aproximadamente, a metade das escritas dos estudantes não refletia o que eles fizeram durante a investigação. Desta forma, os autores perceberam que existe uma lacuna no momento em que os alunos escrevem sobre os procedimentos que realizaram. Uma explicação admissível é a de que os alunos não sabiam que tipo de informações deveriam inserir nas seções referentes às hipóteses, às evidências e às explicações. Outra explicação é de que os alunos não sabiam como expressar os seus modelos mentais em palavras. Nesse sentido, os autores inferem que sistemas tutores inteligentes, baseados em técnicas de scaffolding, poderiam auxiliar os estudantes.

Em outro estudo, procurou-se avaliar o nível de formalidade na escrita de explicações científicas no formato CER (Claim, Evidence, Reasoning) de estudantes do ensino fundamental utilizando a ferramenta de Coh-Metrix (LI et al., 2018). Ela apresenta 5 dimensões que podem ser analisadas em conjunto para mensurar a formalidade de um texto, a saber: concretude das palavras, simplicidade sintática, narratividade, coesão geral e coesão profunda. Ao todo, 293 estudantes das $7^{\mathrm{a}}$ e $8^{\mathrm{a}}$ série do ensino fundamental participaram de um experimento para avaliar a formalidade da escrita condizente a conceitos científicos. No experimento todos os estudantes realizaram atividades por meio do Inq-ITS, um sistema tutor inteligente voltado a laboratórios virtuais para o ensino de ciências. $O$ estudo indicou que os estudantes utilizaram a linguagem acadêmica, conforme indicado por altos níveis de formalidade, quando escreveram sentenças de evidências e de justificativas, mas não em suas hipóteses. Os estudantes também procuraram utilizar sintaxe e textos mais complexos ao construírem suas declarações de CER, porém, a qualidade da coesão textual diminuiu. Desta forma, o estudo mostrou que eles precisam ser instruídos a usar verbos causais ou conectivos causais para especificar explicitamente os relacionamentos em suas explicações. Os alunos, além disso, necessitam ser instruídos a evitar o uso de pronomes vagos para se referirem a uma pessoa ou coisa em sua redação acadêmica formal, pois esse idioma descontextualizado pode levar à falta de clareza. Para os autores, estas lacunas na escrita poderiam ser preenchidas por meio de ferramentas de scaffolding com apoio de sistemas tutores inteligentes.

Por fim, Rapp e Kauf (2018) apresentaram um estudo que buscou avaliar a usabilidade e a utilidade de um sistema chamado de Thesis Writer (TW), uma ferramenta de scaffolding focada em instrução, planejamento e domínio de gênero de trabalhos finais de graduação e artigos no formato IMRD (Introduction, Methods, Results and Discussion). A justificativa para o estudo apontava o grande número de alunos e o alto custo do processo instrucional da escrita. O TW foi projetado para auxiliar na redação de teses por meio de diversas ferramentas e tutoriais, guiando os alunos no processo de redação acadêmica, desde a proposta inicial até a escrita completa da tese. Ainda, o sistema permite que os professores e outros estudantes colaborem, em tempo real, na escrita. Para verificar a usabilidade e a utilidade da ferramenta foi realizado um experimento quali-quantitativo com 102 estudantes do primeiro ano do curso de Administração durante 14 semanas. Dentre as funcionalidades do sistema, duas ganharam evidência nas respostas dos alunos: a organização do processo de scaffolding, o qual permitiu aos alunos 
focarem em pontos específicos, como a questão de pesquisa, por exemplo; e a escrita colaborativa, uma vez que os colegas poderiam auxiliar na escrita dentro da própria ferramenta, ao contrário de enviarem mensagens de e-mail, por exemplo. Além disso, a funcionalidade de phrases book, que consistia em um conjunto de frases pré-definidas para auxiliar os alunos com a escrita acadêmica, foi mencionada como útil pelos participantes do estudo.

Com base na leitura dos documentos selecionados para esta revisão da literatura, foi possível extrair as informações que auxiliaram a responder as questões de pesquisa, conforme apresentado na Tabela 4.

Tabela 4 - Análise quantitativa dos trabalhos.

\begin{tabular}{|c|c|c|c|c|}
\hline Gênero acadêmico & Nível de ensino & Público-alvo & $\begin{array}{c}\text { Objetivo } \\
\text { principal }\end{array}$ & $\begin{array}{c}\text { Nível de } \\
\text { abrangência da } \\
\text { escrita }\end{array}$ \\
\hline $\begin{array}{c}\text { Ensaio (1) } \\
\begin{array}{c}\text { Artigo (1) } \\
\text { Tese (3) }\end{array}\end{array}$ & $\begin{array}{c}\text { Ensino Fundamental } \\
(2)\end{array}$ & & Avaliação (6) \\
$\begin{array}{c}\text { Ensino-médio (2) } \\
\text { Graduaçãa (1) } \\
\text { de curso (1) } \\
\text { Não especificado (2) }\end{array}$ & Alunos (8) & $\begin{array}{c}\text { Aprendizagem } \\
(2)\end{array}$ & $\begin{array}{c}\text { Geral (5) } \\
\text { Específico (3) }\end{array}$ \\
\hline Total (8) & Total (8) & Total (8) & Total (8) & Total (8) \\
\hline
\end{tabular}

Fonte: os autores.

A seguir, são apresentadas as respostas às questões de pesquisa desta revisão sistemática obtidas a partir da análise dos artigos selecionados.

Com relação à QP1 (Para quais gêneros de escrita acadêmica os trabalhos são direcionados?), é possível perceber que a maioria dos trabalhos está relacionada com a escrita da tese. Entretanto, é necessário considerar que eles possuem autores em comum. Além disso, existem propostas, em menor quantidade, focadas em auxiliar os alunos em trabalhos de conclusão de curso, em artigos científicos e em ensaios. Apesar de os pesquisadores, de um modo geral, proporem soluções interessantes para auxiliar os estudantes na produção de diferentes gêneros de escrita acadêmica, ainda há muitas questões não solucionadas. Rigo et al. (2018), por exemplo, destacaram a dificuldade dos estudantes em iniciar a escrita ou, simplesmente, escrever melhor. Algumas das ferramentas propostas buscam sanar alguns destes problemas, como na pesquisa de Lin, Liu e Wang (2017) que utiliza exemplos de sentenças para guiar os estudantes em diferentes momentos da produção textual. Observa-se, no entanto, que tal estratégia pode tornar-se repetitiva e de difícil adaptação para textos em áreas distintas de conhecimento. Neste sentido, é possível apontar uma carência de soluções que tenham capacidade adaptativa e possam lidar com textos em diferentes áreas.

Com relação ao público-alvo dos estudos (QP2), observa-se nos estudos selecionados um total direcionamento aos alunos. Após a identificação desta orientação, tem-se sua segmentação conforme a Tabela 4, onde observa-se uma predominância de estudantes do ensino de pósgraduação, enquanto a minoria é relacionada à graduação. Nesse sentido, é importante ressaltar que é durante a graduação que ocorrem os primeiros contatos dos estudantes com os requintes 
da escrita acadêmica e onde sobressaem-se fatores como a inexperiência com relação às convenções acadêmicas e a heterogeneidade, tanto cultural, como de trajetória escolar da população discente, as quais podem fomentar dificuldades de escrita capazes de gerar entraves que, em alguns casos, mantem-se durante toda a vida acadêmica, inclusive após a graduação (ASSIS, 2014). Ainda, é preciso aceitar que os estudantes universitários familiarizam-se e aprendam a escrever os gêneros acadêmicos quando se inserem nas práticas de escrita na universidade. Logo, além de lidar com a complexidade intrínseca ao processo de escrita, eles precisam estar atentos às convenções específicas de cada gênero acadêmico e, mesmo que tais convenções apresentem um elevado grau de padronização, muitas vezes elas não são claras para quem escreve, e acarreta uma série de problemas durante a escrita, como organização textual ineficiente (FELTRIM, 2004). Assim, esta revisão sistemática aponta para um nicho pouco explorado, contudo, necessário.

Para responder a terceira questão de pesquisa e identificar o objetivo dos trabalhos (QP3), este estudo classificou os artigos pesquisados em duas categorias. A primeira contém todos os trabalhos relacionados à avaliação da escrita (o produto), enquanto a segunda possui os estudos que têm como objetivo apoiar o processo de escrita acadêmica. A partir das publicações selecionadas, percebeu-se que a maioria das propostas mantém o foco na avaliação da escrita fornecidas pelos sistemas, e pouca atenção tem sido direcionada a estratégias para qualificar o processo. Porém, dadas as dificuldades inerentes à atividade de escrita (FELTRIM, 2004), tornase interessante investigar como ferramentas computacionais, especialmente apoiadas por sistemas tutores inteligentes, podem contribuir para a qualidade do texto durante o processo.

Para responder à última questão, os trabalhos foram classificados como sendo de foco geral ou específico no que tange à abrangência de aspectos linguísticos (QP4). Nesse sentido, percebeu-se que 5 projetos focaram em estudar aspectos a escrita em nível macro, por exemplo, a coerência entre seções de um texto, enquanto os outros 3 estudos abordaram pontos mais específicos, como a gramática empregada na escrita de explicações científicas. É necessário considerar, conforme a análise realizada para responder a QP3, que a maioria dos trabalhos é focada na avaliação do produto escrito. Logo, abordagens que envolvam estratégias instrucionais, feedback e suporte em nível macro podem ser um campo fértil de pesquisa.

\section{Considerações Finais}

Este estudo apresentou o resultado de uma revisão sistemática da literatura que teve como objetivo identificar de que forma a utilização de sistemas tutores inteligentes tem contribuído para a processo de escrita acadêmica. Todavia, foi possível perceber que apesar da importância da escrita no âmbito acadêmico e das possibilidades dos STIs, existem poucas pesquisas que buscam relacionar estes dois temas.

Dentre os trabalhos analisados, a maioria é direcionada à escrita de teses, com níveis de abrangência micro (por exemplo, na avaliação da argumentação em determinada seção). Outro aspecto interessante é que eles buscam, em grande parte, alguma forma de avaliação da escrita dos alunos, ao contrário de focar em estratégias para qualificar o processo de escrita acadêmica. 
Desta forma, assemelham-se às funcionalidades de AES (Automated Essay Scoring) e AWS (Automated Writing Evaluation), conforme apresentado por Allen, Jacovina e McNamara (2016).

A partir dos estudos aqui analisados observa-se a necessidade de desenvolvimento de novas pesquisas com vistas a investigar como STIs podem contribuir na qualidade da produção da escrita acadêmica, especialmente no que tange à organização e desenvolvimento de ideias, a principal dificuldade encontrada na escrita (RIGO et al., 2018). Percebeu-se, também, uma lacuna com relação à escrita de projetos de pesquisa, o que constitui, em muitos casos, o primeiro gênero de escrita acadêmica elaborado pelos estudantes e é um dos mais importantes a se observar, tanto por causa de sua relevância social e institucional, quanto pelo conjunto de conhecimentos necessários para a sua elaboração (ARAÚJO; DIEB; COSTA, 2017).

\section{Referências}

ALLEN, L. K.; MILLS, C.; JACOVINA, M. E.; CROSSLEY, S.; D'MELLO, S.; MCNAMARA, D. S. Investigating boredom and engagement during writing using multiple sources of information: The essay, the writer, and keystrokes. In: ACM INTERNATIONAL CONFERENCE PROCEEDING SERIES 2016a, Anais [...]. [s.I: s.n.] p. 114-123. DOI: 10.1145/2883851.2883939. Disponível em: https://www.scopus.com/inward/record.uri?eid=2-s2.084976473432\&doi $=10.1145 \% 2 F 2883851.2883939 \&$ partnerID $=40 \&$ md5=91eda585bf3803756319abbb501 b7969.

ALLEN, Laura K.; JACOVINA, Matthew E.; MCNAMARA, Danielle S.; ALLEN, Laura K.; JACOVINA, Matthew E.; MCNAMARA, Danielle S. Computer-Based Writing Instruction Computer-Based Writing Instruction. [S. I.], 2016. b.

ARAÚJO, Júlio; DIEB, Messias; COSTA, Sayonara Melo. O QNP e as dificuldades de construção do objeto de pesquisa: uma experiência de aprendizagem mediada sobre o gênero projeto de pesquisa. DELTA: Documentação de Estudos em Lingüística Teórica e Aplicada, [S. I.], v. 33, n. 3, p. 729-757, 2017. DOI: $10.1590 / 0102-445097873044367046$.

ASSIS, Juliana Alves. Ações do professor e do universitário nas práticas de ensino e de aprendizagem da escrita acadêmica: o papel da avaliação e da reescrita no processo de apropriação do gênero resenha. Ações do professor e do universitário nas práticas de ensino e de aprendizagem da escrita acadêmica: o papel da avaliação e da reescrita no processo de apropriação do gênero resenha, [S. I.], v. 1, n. 13, p. 543-561, 2014. DOI: 10.19134/eutomia-v1i13p543-561.

FARIAS, Sandra Alves; BORTOLANZA, Ana Maria Esteves. 0 papel da linguagem escrita nos processos de ensino e aprendizagem: um estudo teórico. Educação \& Linguagem, v. 18, n. 2, p. 63-85, 2015.

FELTRIM, V. D. Uma abordagem baseada em córpus e em sistemas de crítica para a construção de ambientes web de auxílio à escrita acadêmica em português. [S. I.], 2004.

GARCÍA-GORROSTIETA，J. M.; LÓPEZ-LÓPEZ, A.; GONZÁLEZ-LÓPEZ, S. Towards automatic assessment of argumentation in theses justifications. Lecture Notes in Computer Science (including subseries Lecture Notes in Artificial Intelligence and Lecture Notes in Bioinformatics), [S. I.], 2017. DOI: 10.1007/978-3-319-66610-5_5. Disponível em: https://www.scopus.com/inward/record.uri?eid=2-s2.0$85029575841 \&$ doi $=10.1007 \% 2$ F978-3-319-66610-

5_5\&partnerID $=40 \& m d 5=d 3 c d 55 e 1 e e 50 b 1 f e 28 f 9 e 1 b e f c 3 f 502 c$.

HIEU, B. T.; MUSTAPHA, S. M. F. D. S. Automated data-driven hint generation in intelligent tutoring systems for code-writing: On the road of future research. International Journal of Emerging Technologies in Learning, [S. I.], v. 13, n. 9, p. 174-189, 2018. DOI: 10.3991/ijet.v13i09.8023. Disponível em: https://www.scopus.com/inward/record.uri?eid=2-s2.0-

85057574543\&doi=10.3991\%2Fijet.v13i09.8023\&partnerID=40\&md5=349e90c4f60f6f19a18e4cd048a724 $6 \mathrm{~b}$.

KITCHENHAM, Barbara. Procedures for performing systematic reviews. Keele, UK, Keele University, [S. I.], v. 33, n. TR/SE-0401, p. 28, 2004. DOI: 10.1.1.122.3308. Disponível em: http://csnotes.upm.edu.my/kelasmaya/pgkm20910.nsf/0/715071a8011d4c2f482577a700386d3a/\$FILE/10 .1.1.122.3308[1].pdf\%5Cnhttp://tests-zingarelli.googlecode.com/svn-history/r336/trunk/2- 
Disciplinas/MetodPesquisa/kitchenham_2004.pdf.

LI, H.; GOBERT, J.; DICKER, R. Dusting off the messy middle: Assessing students' inquiry skills through doing and writing. Lecture Notes in Computer Science (including subseries Lecture Notes in Artificial Intelligence and Lecture Notes in Bioinformatics), [S. I.], 2017. a. DOI: 10.1007/978-3-319-614250_15. Disponível em: https://www.scopus.com/inward/record.uri?eid=2-s2.085022191215\&doi $=10.1007 \% 2 \mathrm{~F} 978-3-319-61425-$

0_15\&partnerID=40\&md5=ebc40c130f3ce82a4196b0128836651d.

LI, H.; GOBERT, J.; DICKER, R. Dusting off the messy middle: Assessing students' inquiry skills through doing and writing. Lecture Notes in Computer Science (including subseries Lecture Notes in Artificial Intelligence and Lecture Notes in Bioinformatics), [S. I.], v. 10331 LNAI, p. 175-187, 2017. b. DOI: 10.1007/978-3-319-61425-0_15. Disponível em: https://www.scopus.com/inward/record.uri?eid=2-s2.085022191215\&doi $=10.1007 \% 2$ F978-3-319-61425-

$0 \_15 \&$ partnerID =40\&md5=ebc40c130f3ce82a4196b0128836651d

LI, H.; GOBERT, J.; DICKLER, R.; MORAD, N. Students' academic language use when constructing scientific explanations in an intelligent tutoring system. Lecture Notes in Computer Science (including subseries Lecture Notes in Artificial Intelligence and Lecture Notes in Bioinformatics), [S. I.], 2018. DOI: 10.1007/978-3-319-93843-1_20. Disponível em: https://www.scopus.com/inward/record.uri?eid=2-s2.0$85049376471 \&$ doi $=10.1007 \% 2 F 978-3-319-93843-$

1_20\&partnerID $=40 \&$ md5=8625b883db58beebc209755098639a83.

LIN, C. C.; LIU, G. Z.; WANG, T. I. Development and usability test of an e-learning tool for engineering graduates to develop academic writing in English: A case study. Educational Technology and Society, [S. l.], 2017. Disponível em: https://www.scopus.com/inward/record.uri?eid=2-s2.085032219092\&partnerID =40\&md5=8b491d603bcaa1aeba608289f8244369.

LÓPEZ, S. G.; BETHARD, S.; LÓPEZ-LÓPEZ, A. Identifying Weak Sentences in student drafts: A tutoring system. Advances in Intelligent Systems and Computing, [S. I.], 2014. DOI: 10.1007/978-3-31907698-0_10. Disponível em: https://www.scopus.com/inward/record.uri?eid=2-s2.084905675640\&doi $=10.1007 \% 2$ F978-3-319-07698-

$0 \_10 \&$ partnerID $=40 \&$ md5 $=5$ cadc563682dfff34a0abea2f071bc8c.

LÓPEZ, S. G.; LÓPEZ-LÓPEZ, A. Mining domain knowledge for coherence assessment of students proposal drafts. Studies in Computational Intelligence, [S. I.], 2014. DOI: 10.1007/978-3-319-02738-8_9, Disponível em: $84958536346 \&$ doi $=10.1007 \% 2 F 978-3-319-02738$ -

8_9\&partnerID $=40 \& \mathrm{md5}=47 \mathrm{fc} 1 \mathrm{edcb} 2 \mathrm{e} 3062 \mathrm{a} 67008 \mathrm{f} 18 \mathrm{e} 715 \mathrm{~d} 992$.

NWANA, Hyacinth S. Intelligent tutoring systems: an overview. Artificial Intelligence Review, [S. I.], v. 4, n. 4, p. 251-277, 1990. DOI: 10.1007/BF00168958.

RAPP, C.; KAUF, P. Scaling Academic Writing Instruction: Evaluation of a Scaffolding Tool (Thesis Writer). International Journal of Artificial Intelligence in Education, [S. I.], 2018. DOI: 10.1007/s40593-0170162-z. Disponível em: https://www.scopus.com/inward/record.uri?eid=2-s2.0$85055053925 \&$ doi $=10.1007 \% 2 F s 40593-017-0162-$

z\&partnerID $=40 \& m d 5=635 c 09 d 069 a b 76567 c 926 b 57 b 53 e 6987$.

REATEGUI, Eliseo Berni; OLIVEIRA, Simone D. E. O Apoio de Uma Ferramenta com Base Na Mineração De Texto Para Escrita Acadêmica Support of a Tool Based on Text Mining for Academic Writing. [S. I.], p. 3645, 2017.

REATEGUI, Eliseo; EMER, Simone de Oliveira De; MAUER, Jocimara de Lima; GOMES, André; DAPPER, Leandro Leonel. Mineração De Texto No Apoio Da Escrita Acadêmica. Revista Novas Tecnologias na Educação (RENOTE), [S. I.], v. 12, n. 2, p. 2-11, 2014. Disponível em: http://seer.ufrgs.br/index.php/renote/article/view/53537.

RIGO, Rosa Maria; COSTA, Fábio Soares Da; RAMIREZ, Rosa Eulógia; VITÓRIA, Maria Inês Côrte. Escrita acadêmica: fragilidades, potencialidades e articulações possíveis | Academic writing: Weaknesses, potentialities and possible associations. Revista de Educação PUC-Campinas, [S. I.], v. 23, n. 3, p. 489, 2018. DOI: $10.24220 / 2318-0870 v 23 n 3 a 3952$.

STROBL, Carola; AILHAUD, Emilie; BENETOS, Kalliopi; DEVITT, Ann; KRUSE, Otto; PROSKE, Antje; RAPP, Christian. Digital support for academic writing: A review of technologies and pedagogies. Computers and Education, [S. I.], v. 131, p. 33-48, 2019. DOI: 10.1016/j.compedu.2018.12.005. Disponível em: https://www.scopus.com/inward/record.uri?eid=2-s2.0-

85059147392\&doi $=10.1016 \% 2 F j . c o m p e d u .2018 .12 .005 \&$ partnerID $=40 \& \mathrm{md} 5=\mathrm{c} 8 \mathrm{e} 3 \mathrm{c} 7 \mathrm{e} 83547 \mathrm{da} 09 \mathrm{f} 1 \mathrm{fe} 9234$ 
4003 fef3.

WESTON-SEMENTELLI, Jennifer L.; ALLEN, Laura K.; MCNAMARA, Danielle S. Comprehension and Writing Strategy Training Improves Performance on Content-Specific Source-Based Writing Tasks. International Journal of Artificial Intelligence in Education, [S. I.], v. 28, n. 1, p. 106-137, 2018. DOI: 10.1007/s40593-016-0127-7.

Recebido em junho de 2020

Aprovado para publicação em janeiro de 2021

\section{Ederson Bastiani}

Programa de Pós-Graduação em Informática da Educação - Universidade Federal do Rio Grande do Sul UFRGS, Brasil, edersonbastiani@gmail.com

\section{Alex Eder da Rocha Mazzuco}

Programa de Pós-Graduação em Informática da Educação - Universidade Federal do Rio Grande do Sul UFRGS, Brasil, edersonbastiani@gmail.com

\section{Eliseo Berni Reategui}

Faculdade de Educação da Universidade Federal do Rio Grande do Sul e Programa de Pós-Graduação em Informática na Educação - Universidade Federal do Rio Grande do Sul - UFRGS, Brasil, eliseoreategui@gmail.com 